\title{
VARIABILIDADE TEMPORAL DA RADIAÇÃO SOLAR E INSOLAÇÃO NO MUNICÍPIO DE ARAGUAÍNA - TO'
}

\section{Temporal variability of the solar radiation and insolation in Araguaína - TO}

\author{
Dóris Macêdo dos SANTOS² \\ Silvânia Moreira de ARAÚJO² \\ Kallianna Dantas ARAUJO3 \\ Paulo Roberto de Oliveira ROSA ${ }^{4}$ \\ Eduardo PAZERA JR. ${ }^{5}$
}

\begin{abstract}
RESUMO
O objetivo da pesquisa foi avaliar a variabilidade temporal da radiação solar e insolação no município de Araguaína - TO. Os dados meteorológicos (isolação e nebulosidade) foram obtidos da estação convencional do INMET, localizada no campus de Medicina Veterinária e Zootecnia. A radiação solar global $(\mathrm{Qg})$ foi obtida por meio de imagens de satélite GOES e a partir desta foi calculado o total da radiação solar refletida ( $\mathrm{Qr}$ ) e absorvida (Qa). O albedo estabelecido foi de $0,3 \%$ de refletividade. As maiores incidências da $\mathrm{Qg}$ e da insolação foram verificadas nos meses de abril, maio, junho, julho, agosto e setembro, coincidindo com o período de menor nebulosidade local; A amplitude de variação anual da nebulosidade na região é relativamente elevada, variando de 1 a $8 / 10$ ao longo do ano.
\end{abstract}

Palavras-chave: Clima; Variabilidade; Radiação solar.

\begin{abstract}
This paper aimed at evaluating the temporal variability of the solar radiation and insolation in Araguaína-TO. The meteorological data (insolation and cloudiness) had been gotten of the conventional station of the INMET, located in the campus of "Medicina Veterinária e Zootecnia". The global solar radiation $(\mathrm{Qg})$ was gotten by means of images of satellite GOES and from this solar radiation total reflected (Qr) and absorbed was calculated (Qa). The established Albedo was of $0,3 \%$ of reflectivity. The biggest incidences of the $\mathrm{Qg}$ and the insolation had been verified in the months of April, May, June, July, August and September, coinciding with the period of lesser local cloudiness. The amplitude of annual variation of the cloudiness in the region is relatively high, varying from 1 to $8 / 10$ throughout the year.
\end{abstract}

Keywords: Climate; Variability; Solar radiation. \footnotetext{
Araguaína.

1 Parte do Trabalho de Conclusão de Curso apresentado ao Curso de Geografia da Universidade Federal do Tocantins, Campus de

2 Aluna do Curso de Geografia da Universidade Federal do Tocantins, Campus de Araguaína. E-mail: doris01-macedo@yahoo.com. br, smaraujo78@yahoo.com.br

3 Geógrafa. MSc. Doutoranda do PPGRN/CTRN/UFCG. E-mail: kdaraujo@yahoo.com.br

4 Professor Adjunto Depto. de Geociências, CCEN, UFPB. E-mail: paulorosa_ufpb@hotmail.com

5 Geógrafo. Dr. SINAES -MEC. E-mail: pazera@terra.com.br
} 
SANTOS, D. M. dos et al. Variabilidade temporal da radiação solar e insolação no...

\section{INTRODUÇÃO}

Os ambientes tropicais caracterizam-se pela elevada incidência de radiação solar, insolação e temperatura. Quanto mais próximo do equador, mais crítica é a incidência, como é o caso das regiões norte, nordeste e centro-oeste do Brasil (BAYER, 2004; BLEY JR., 1999).

A radiação solar incidente sobre a superfície do solo é fator determinante e condiciona os processos que ocorrem nesse ambiente, interferindo sobremaneira no ciclo dos nutrientes. Nestes termos, variando a quantidade de radiação solar incidente, podem-se obter condições ambientais diferenciadas e, por conseguinte, promover alteração nos demais processos (ARAUJO, 2005; SILVA et al., 1999).

Os efeitos diretos que a incidência diferenciada da radiação solar causa na superfície do solo podem ser constatados através da determinação da temperatura e umidade deste. A intensidade da radiação solar define as condições microclimatológicas, a temperatura sendo diretamente proporcional à radiação solar, enquanto a umidade se relaciona inversamente (SILVA et al., 1999).

Estudos relativos à disponibilidade de totais diários médios mensais da irradiação solar global são importantes no planejamento agrícola, uma vez que esta variável é fundamental para a determinação do saldo de radiação, bem como, para a estimativa da evapotranspiração de uma determinada cultura (SOUZA et al., 2005). De forma complementar Silva et al. (1999) afirmam que a radiação solar e a temperatura são os dois principais fatores que influenciam na perda de água do solo.

Os solos presentes nas regiões tropicais são, regra geral, altamente intemperizados e têm suas condições químicas, físicas e biológicas altamente dependentes da matéria orgânica o que determina uma grande resposta destes solos a sistemas de manejo que promovem uma variação positiva nos estoques de C (Carbono) orgânico do solo (BAYER, 2004). Quando os solos ficam expostos à intensa radiação solar e às altas temperaturas, ocorre a oxidação de sua matéria orgânica (SHAXSON, 1988).

É nesse sentido que Bley Jr. (1999) menciona que para produzir em solos tropicais é necessário aprofundar o conhecimento sobre os fatores característicos dessas regiões, como os efeitos do Sol, que a ciência desenvolvida em regiões de clima temperado e frio não considera, ou trata apenas como coadjuvantes das erosões hídrica e eólica.
O objetivo da pesquisa foi avaliar a variabilidade temporal da radiação solar e insolação no município de Araguaína - TO.

\section{MATERIAL E MÉTODOS}

O trabalho foi desenvolvido no município de Araguaína - TO, cuja posição geográfica corresponde às coordenadas de $07^{\circ} 11^{\prime} 28^{\prime \prime}$ S e 48 $12^{\circ} 26^{\prime \prime} \mathrm{W}$, com altitude de 277 m (IBGE, 2005). Apresenta um relevo com formas estruturais (Superfície Tabulares Estruturais e Patamares Estruturais), com predominância de áreas com declive suave igual ou superior a $5 \%$. Do ponto de vista geológico faz parte da Bacia Sedimentar do Parnaíba, com compartimentação geoambiental do tipo Domínio das Bacias Sedimentares Paleo-Mesozoicas e MesoCenozoicas (Depressões e Patamares de Ananás e Araguaína). Possui solo do tipo Latossolos e Neossolos Quartzarênicos (EMBRAPA, 1999). A vegetação encontrada é do tipo Cerrado (GOVERNO DO ESTADO DO TOCANTINS, 2003). A rede hidrográfica é formada pelo rio Araguaia e seus afluentes (SANTIAGO, 2000).

Apresenta o clima úmido do tipo B1wA'a' segundo a classificação de Thornthwaite com deficiência hídrica moderada. A precipitação média anual varia entre 1700 a $1800 \mathrm{~mm}$ e a temperatura média anual é $28^{\circ} \mathrm{C}$, com máxima de $32{ }^{\circ} \mathrm{C}$ e a mínima de $20^{\circ} \mathrm{C}$.

Os dados meteorológicos (isolação e nebulosidade) foram obtidos da estação convencional pertencente ao $10^{\circ}$ Distrito de Meteorologia do Instituto Nacional de Meteorologia (INMET) localizada a $7^{\circ} 20^{\prime}$ 'S e $48^{\circ} 7^{\prime} \mathrm{W}$ na altitude de $228,52 \mathrm{~m}$ a $15 \mathrm{~km}$ da sede municipal no campus de Medicina Veterinária e Zootecnia. Os dados de radiação solar global foram obtidos por meio de imagens de satélite GOES (INPE, 2005).

Calculou-se o total da radiação solar refletida e a absorvida por uma superfície específica, a partir dos dados de radiação global do Sol, estabelecendo um processo comparativo entre os três níveis mediante a metodologia utilizada por D'Angiolella et al. (2001). Os cálculos da radiação solar refletida (Qr) e da absorvida (Qa) foram feitos mediante a aplicação da fórmula de Tubelis e Nascimento (1988), onde $Q r=r$. Qg ao passo que $Q a=(1-r)$. $Q g$, com $r$ representando o albedo da superfície e $\mathrm{Qg}$ é a radiação solar global. O albedo estabelecido foi de $0,3 \%$ de refletividade (ARAUJO, 2005). 


\section{RESULTADOS E DISCUSSÃO}

Verificou-se que durante o período de setembro de 2002 a agosto de 2005 a radiação solar global foi maior que absorvida e refletida para todos os meses analisados (Figura 1) estando de acordo com os dados encontrados por D'Angiolella et al. (2001) e Araujo (2005). Segundo Geiger (1961) ao longo do dia, nas horas de brilho solar, o saldo de radiação em uma superfície qualquer tende a ser positivo, pois os fluxos, incidente global e atmosférico são superiores às frações refletidas e emitidas. Durante a noite, é comum que esses valores sejam negativos, pois o fluxo incidente passa a ser apenas atmosférico e a energia emitida pela superfície, superior a este, o que resulta em um saldo de radiação negativa.

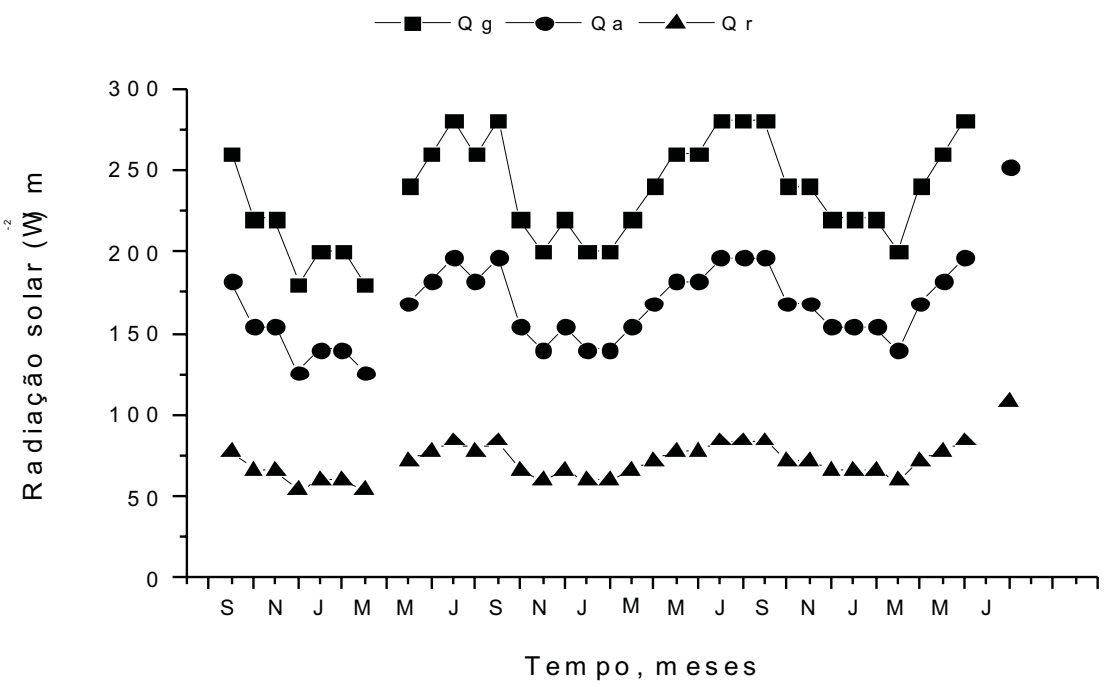

FIGURA 1 - RADIAÇÃO SOLAR GLOBAL (QG), RADIAÇÃO ABSORVIDA (QA) E RADIAÇÃO REFLETIDA (QR) DE ARAGUAÍNA - TO, REFERENTES AO PERÍODO DE SETEMBRO DE 2002 A JUNHO DE 2005

A radiação solar foi elevada no período abrilsetembro atingindo $280,0 \mathrm{~W} \mathrm{~m}^{-2}$ nos meses de julho e setembro (2003) e julho a setembro (2004), que caracteriza a época seca da região, decorrente da reduzida nebulosidade na área e consequentemente do maior número de horas de insolação. O período dezembromarço apresentou menor radiação solar incidente, atingindo $180,0 \mathrm{~W} \mathrm{~m}^{-2}$ em dezembro (2003) e março (2004), na época chuvosa. D'Angiolella et al. (2001) estudando as características da radiação e suas aplicações no Recôncavo Baiano também verificaram que a radiação solar foi elevada no período seco da região, que compreende os meses entre dezembro e março. Padrões de radiação comparativamente inferiores ocorreram entre abril a setembro, no período chuvoso.

De forma complementar, Silva et al. (2005) constataram que a radiação solar média mensal apresentouse mais alta nos meses de primavera-verão, atingindo o máximo no mês de dezembro e o mínimo em junho. Sacco e Assis (2005) estudando a estimativa da radiação solar global em dias com nuvens para a região de Pelotas-RS, constataram uma distribuição sazonal bem definida com valores máximos no verão e mínimos no inverno, acompanhando a variação anual imposta pela movimentação solar.

Silva et al. (1999) verificaram diferenças quanto à incidência dos raios solares provocada pela variação da declividade e exposição do terreno e, também pela época do ano; onde observou-se que as rampas com exposição sul receberam menor quantidade de radiação solar que as rampas com exposição norte, principalmente no inicio do experimento, de julho a setembro, e maior quantidade de radiação solar no período de setembro a dezembro.

No município de Araguaína - TO o número de horas de insolação totaliza 2.400 horas anuais e 200 horas mensais, atingindo 6,5 horas diárias (INMET, 2005) (Figura 2). Nesse sentido, embora o município esteja localizado próximo ao equador, a insolação diária é sempre inferior ao comprimento do dia, uma vez que o número de horas de nebulosidade do município apresenta uma média anual de 6/10 (INMET, 2005) (Figura 3). 


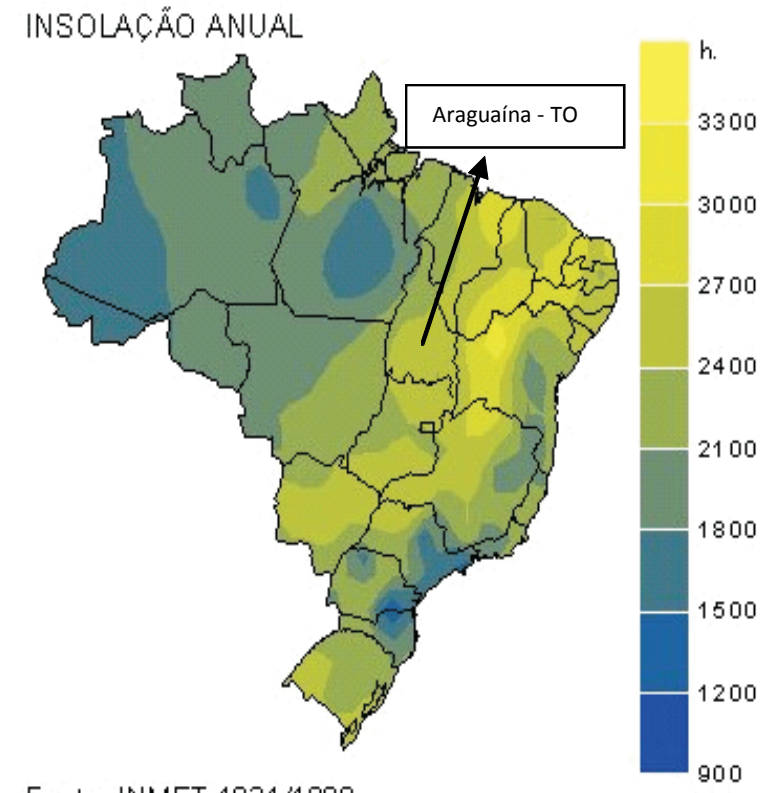

Fonte: INMET 1931/1990

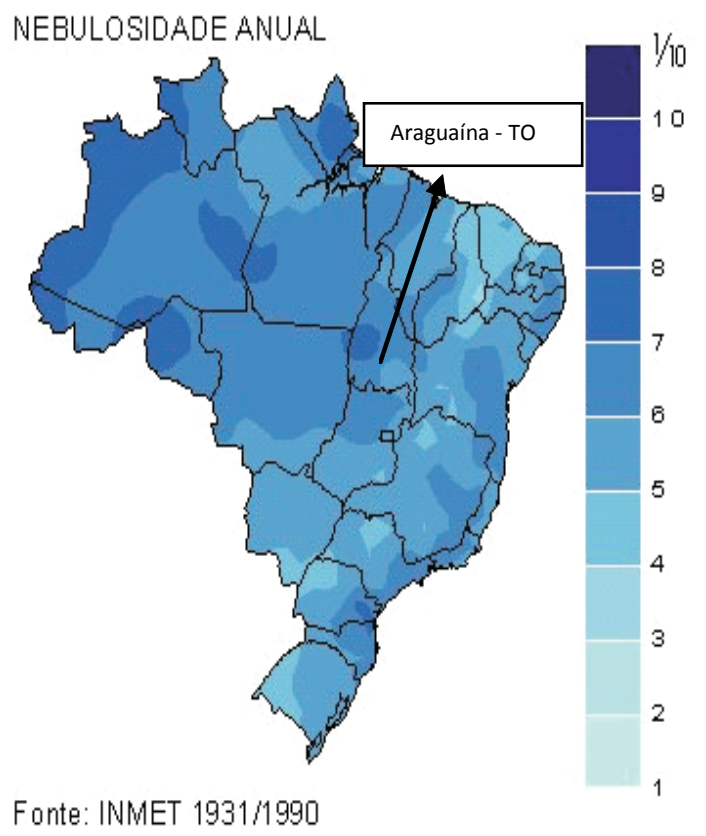

FIGURA 3 - NEBULOSIDADE MÉDIA ANUAL DE ARAGUAÍNA - TO
No Brasil, verifica-se em termos médios anuais, que o céu fica encoberto de 24 a $52 \%$ do período diurno e que a insolação é maior que a cobertura do céu de janeiro a abril na região Sul, de junho a outubro na região Norte, de abril a janeiro na região Nordeste, de março a janeiro na região Sudeste e durante o ano todo na região Centro-Oeste, totalizando uma média anual de insolação entre 1.600 e 3.200 horas (TUBELIS; NASCIMENTO, 1988).

Mota (1983) considera que $1 \mathrm{cal} \mathrm{cm}^{-2} \mathrm{~min}^{-1}$ representa aproximadamente uma evaporação de $1 \mathrm{~mm}$ de água por hora. Na área em estudo o total de evaporação é de 1200 a $1600 \mathrm{~mm}$ anuais (INMET, 2005). Cunha et al. (2005) indicam que a densidade do dossel tem grande importância no regime de radiação solar, sendo importante sua caracterização para o entendimento dos processos que governam a dinâmica do ecossistema florestal. Os mesmos autores observaram uma atenuação da radiação solar pelo dossel de floresta nativas em regiões tropicais. De forma complementar Galvani et al. (2001) mencionam que quando se forma um dossel homogêneo da cobertura vegetal, observam-se pequenas variações dos valores de FCS devido ao sombreamento do solo, diminuindo os níveis de radiação, verificados pelas curvas de índice de área foliar.

Com relação aos valores de nebulosidade, constatou-se, durante o período de janeiro de 2001 a junho de 2005, que a época de maior nebulosidade foi a que apresentou menor insolação conforme ocorreu no período de outubro-abril, coincidido com o período chuvoso da região. Consequentemente o período da região, de maio-setembro apresentou menor nebulosidade e maior insolação, no período seco da região (Figura 4). Segundo D'Angiolella et al. (2001) o alto teor de umidade e a grande concentração na atmosfera de núcleos de condensação condicionam a formação da nebulosidade reduzindo significativamente a insolação. 


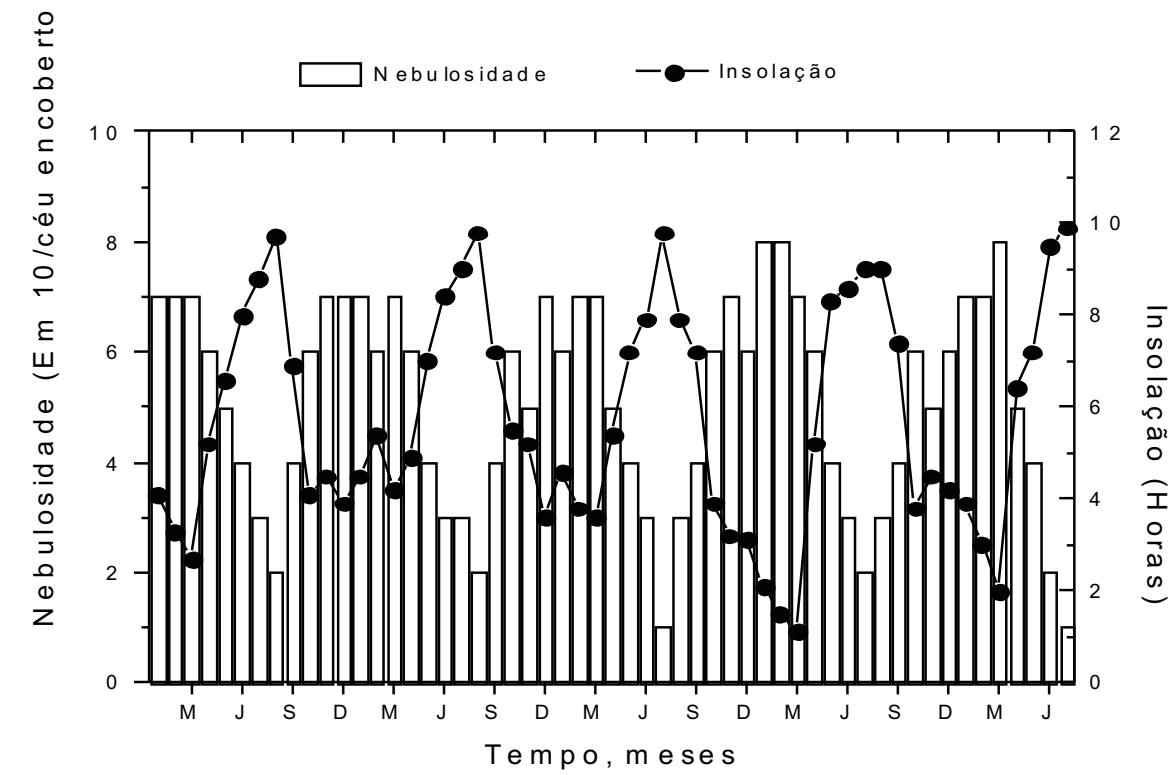

FIGURA 4 - NEBULOSIDADE (EM 10/CÉU) E INSOLAÇÃO (H) DE ARAGUAÍNA - TO, REFERENTES AO PERÍODO DE JANEIRO DE 2001 A JULHO DE 2005

Cunha et al. (2005) verificaram que em determinados dias, houve uma diminuição acentuada nos valores de Rg no campo, aproximando-se dos valores encontrados em ambiente protegido. A causa desse fato é a ocorrência de dias com céu nublado que diminuem a quantidade de $\mathrm{Rg}$ em ambos os ambientes. De forma complementar Cunha et al. (2005) verificaram que a predominância de radiação difusa em dias nublados, tem característica multidirecional e de baixa energia, limitando a ocorrência de picos acentuados, como os observados nos dias com céu limpo.

Observando os dados de energia incidente e nebulosidade durante o período (setembro de 2002 a julho de 2005), constatou-se que a energia incidente foi maior durante o período abril-setembro (Figura 5) Consequentemente a nebulosidade foi menor nesses meses.

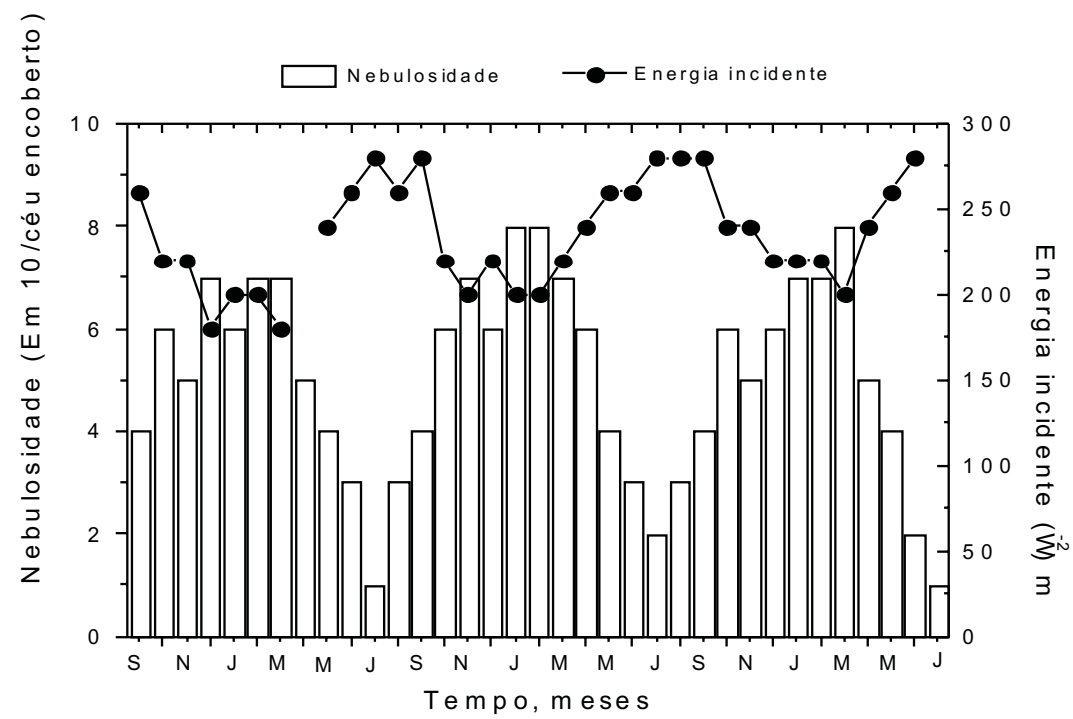

FIGURA 5 - ENERGIA INCIDENTE (W M-2) E NEBULOSIDADE (EM 10/CÉU) DE ARAGUAÍNA - TO, REFERENTES AO PERÍODO DE SETEMBRO DE 2002 A MAIO DE 2005 
SANTOS, D. M. dos et al. Variabilidade temporal da radiação solar e insolação no...

\section{CONCLUSÕES}

- A maior incidência da radiação solar global e insolação foram verificadas nos meses de abril, maio, junho, julho, agosto e setembro, coincidindo com o período de menor nebulosidade local;

- Aamplitude de variação anual da nebulosidade na região é relativamente elevada, variando de 1 a $8 / 10$ ao longo do ano;

\section{REFERÊNCIAS}

ARAUJO, K. D. Variabilidade temporal das condições climáticas sobre as perdas de $\mathrm{CO}_{2}$ na encosta do açude Namorados, em São João do Cariri-PB. 101f. Dissertação (Mestrado em Manejo e Conservação do Solo e Água) - Centro de Ciências Agrárias, Universidade Federal da Paraíba, Areia, 2005.

BAYER, C. Manejando os solos agrícolas para alta qualidade em ambientes tropicais e subtropicais. FERTBIO, 26., 2004, Lages. Anais... Lages: UDESC e Sociedade Brasileira de Ciência do Solo, 2004. CD-ROM.

BLEY JR., C. Erosão Solar: riscos para a agricultura nos trópicos. Ciência Hoje. v. 25, n. 148, p. 24-29, abr. 1999.

CUNHA, A. R. da; KATZ, L.; SOUSA, A. de P.; RODRIGUES, J. D. Caracterização dos níveis de radiação solar no crescimento de plantas de lisianthus cultivados em vasos em ambiente protegido. In: CONGRESSO BRASILEIRO DE AGROMETEOROLOGIA, 14., 2005. Campinas. Anais... Campinas: SBAgro, 2005. CD-ROM.

CUNHA, E. M.; SANTOS, E. A.; PEZZOPANE, J. E. M. BARBOSA, V. S. SILVA, G. F. Caracterização da radiação solar fotossinteticamente ativa em fragmento de floresta estacional semidecidual na bacia do rio Itapemirim, ES. In: CONGRESSO BRASILEIRO DE AGROMETEOROLOGIA, 14., 2005. Campinas. Anais... Campinas: SBAgro, 2005. CD-ROM.

D’ANGIOLELLA, G.; VASCONCELLOS, V. L. D.; SILVA, José de Fátima da; SANCHES, André V. de. Radiação: estudo das características e suas aplicações no Recôncavo Baiano. In: CONGRESSO BRASILEIRO DEAGROMETEOROLOGIAE 12 REUNIÃO LATINO-AMERICANADEAGROMETEOROLOGIA. 3., Fortaleza. Anais... Fortaleza: SBA/FUNCEME, 2001. p. 135.

EMBRAPA. Centro Nacional de Pesquisa de Solos (Rio de Janeiro, RJ). Sistema brasileiro de classificação de solos. Brasília: Produção de Informação. Rio de Janeiro: Embrapa Solos, 1999. 412p.

GALVANI, E.; ESCOBEDO, J. F.; PEREIRA, A. B. Balanço de radiação e fluxo de calor no solo em ambiente natural e protegido cultivado com pepineiro. Bragantia, Campinas, v. 60, n. 2, p. 139-147, 2001.

GEIGER, R. Manual de microclimatologia: o clima da camada de ar junto ao solo. Trad. de Ivone Nogueira e Francisco Caldeira Cabral. Lisboa: Fundação Calouste Gulbenkian, 1961.

GOVERNO DO ESTADO DO TOCANTINS. Atlas do Tocantins: Subsídios ao planejamento da gestão territorial. Secretaria do planejamento e do meio ambiente, Diretoria de zoneamento ecológico-econômico - DZE. 3. ed. Palmas: Seplan, 2003. $49 p$.

INSTITUTO BRASILEIRO DE GEOGRAFIAE ESTATÍSTICA. Disponível em: <http://www.ibge.gov.br/cidadesat/default. php>. Acesso em: Setembro 2005.

INSTITUTO DE PESQUISAS ESPACIAIS. Disponível em: <http://satelite.cptec.inpe.br/htmldocs/radiacao/radsol/portal/ radiacao_new.htm>. Acesso em: Julho 2005.

INSTITUTO NACIONAL DE METEOROLOGIA. Disponível em: <http://www.inmet.gov.br/climatologia/combo_climatologia_C. html>. Acesso em: Outubro 2005.

SACCO, F. G.; ASSIS, S. V. Modelo para estimativa da radiação solar global em dias com nuvens para a região de Pelotas-RS. In: CONGRESSO BRASILEIRO DE AGROMETEOROLOGIA, 14., 2005. Campinas. Anais... Campinas: SBAgro, 2005. CD-ROM.

SANTIAGO, C. Araguaína: história e atualidade. Araguaína: Prefeitura Municipal de Araguaína, 2000. 80p.

SHAXSON, T. F. Produção e proteção integradas em microbacias. In: MONIZ, A. C.; FURLANI, A. M. C.; FURLANI, P. R.; FREITAS, S. S. In: CONGRESSO BRASILEIRO DE CIÊNCIA DO SOLO. Campinas, SBCS, 1988, p. 526.

SILVA, J. B. da; LIOPAT, M. P.; BOIASKI, N. T. Análise estatística da radiação solar média mensal em Pelotas-RS. In: CONGRESSO BRASILEIRO DE AGROMETEOROLOGIA, 14., 2005. Campinas. Anais... Campinas: SBAgro, 2005. CD-ROM.

SILVA, M. S.; COSTA, L. A. de M.; BENINCASA, M.; LUCAS JR.; J de. Avaliação das perdas de nitrato em solo irrigado e submetido a diferentes intensidades de radiação solar. Revista Brasileira de Engenharia Agrícola e Ambiental, Campina Grande, v. 3, n. 1, p. 82-88, 1999.

SOUZA, M. J. H.; RIBEIRO, A.; LEITE, F. P.; GOIS, G. Avaliação do modelo de Bristow \& Campbell na estimativa, média mensal dos totais diários da irradiação solar global para o Vale do Rio Doce, MG. In: CONGRESSO BRASILEIRO DE AGROMETEOROLOGIA, 14., 2005. Campinas. Anais... Campinas: SBAgro, 2005. CD-ROM.

TUBELIS, A.; NASCIMENTO, F. J. S. Meteorologia descritiva: fundamentos e aplicações brasileiras. São Paulo: Nobel, 1988. 374p. 